\title{
The Fourier Decomposition for a Radiative-Transfer Problem with an Asymmetrically Reflecting Ground
}

\author{
L. B. Barichello, ${ }^{\dagger}$ R. D. M. Garcia ${ }^{\ddagger}$ and C. E. Siewert ${ }^{\S}$ \\ ${ }^{\dagger}$ Instituto de Matemática \\ Universidade Federal do Rio Grande do Sul \\ 91509-900 Porto Alegre, RS \\ Brasil \\ ${ }^{\ddagger}$ Centro Técnico Aeroespacial \\ Instituto de Estudos Avançados \\ 12231-970 São José dos Campos, SP \\ Brasil \\ $\S_{\text {Center for Research in Scientific Computation }}$ \\ Mathematics Department \\ North Carolina State University \\ Raleigh, NC 27695-8205
}

USA

\begin{abstract}
Elementary considerations are used to carry out a Fourier decomposition of the radiation intensity for a model atmosphere that has a ground defined by a general reflection function. In contrast to simpler problems, the intensity here has both sine and cosine components that are coupled by way of the boundary conditions at the ground.
\end{abstract}

November 28, 1995 


\section{Introduction}

In a recent paper,${ }^{1}$ Godsalve carried out a Fourier analysis for an anisotropically scattering atmosphere illuminated by an incident solar beam. Interestingly, in order to represent better a remote-sensing problem, Godsalve allowed the ground to reflect in an asymmetrical manner, and this ground condition is such that both sine and cosine components are required in a Fourier decomposition of the radiation intensity. However, in reading the paper of Godsalve, we have discovered that serious errors were made in that work in regard to the coupling, by way of the boundary condition at the ground, between the sine and cosine components of the solution. In this work, we report what we believe to be a correct Fourier decomposition that can be used to solve this new class of radiative-transfer problems. We note also that Godsalve introduced the discreteordinates approximation at the beginning of his analysis, and so a set of well-posed component problems that can be used with other solution techniques is not readily available from his paper. Thus here for a general model of ground reflectance, we develop a complete formulation, for all of the component problems related to a Fourier decomposition of the radiation intensity, that is independent of the solution technique to be used.

We let $I(\tau, \mu, \varphi)$ denote the intensity (radiance) of the radiation field and utilize the equation of transfer ${ }^{2}$ for a plane-parallel medium to model our atmosphere. We write

$$
\mu \frac{\partial}{\partial \tau} I(\tau, \mu, \varphi)+I(\tau, \mu, \varphi)=\frac{\varpi}{4 \pi} \int_{-1}^{1} \int_{0}^{2 \pi} p(\cos \Theta) I\left(\tau, \mu^{\prime}, \varphi^{\prime}\right) d \varphi^{\prime} d \mu^{\prime}
$$

where $\tau \in\left(0, \tau_{0}\right)$ is the optical variable and $\varpi$ is the albedo for single scattering. In addition, $\mu \in[-1,1]$ and $\varphi \in[0,2 \pi]$ are, respectively, the cosine of the polar angle (as measured from the positive $\tau$ axis) and the azimuthal angle which describe the direction of propagation of the radiation. We note also that the phase function $p(\cos \Theta)$ is represented by a finite Legendre expansion in terms of the scattering angle $\Theta$, viz.

$$
p(\cos \Theta)=\sum_{l=0}^{L} \beta_{l} P_{l}(\cos \Theta)
$$

where the coefficients are such that $\beta_{0}=1$ and $\left|\beta_{l}\right|<2 l+1$.

We assume that the atmosphere is illuminated uniformly by a solar beam with a direction specified by $\left(\mu_{0}, \varphi_{0}\right)$, and so we seek a solution to Eq. (1) that satisfies the boundary conditions

$$
I(0, \mu, \varphi)=\pi \delta\left(\mu-\mu_{0}\right) \delta\left(\varphi-\varphi_{0}\right)
$$

and

$$
I\left(\tau_{0},-\mu, \varphi\right)=\int_{0}^{2 \pi} \int_{0}^{1} R\left(\mu^{\prime}, \mu, \varphi^{\prime}, \varphi\right) I\left(\tau_{0}, \mu^{\prime}, \varphi^{\prime}\right) \mu^{\prime} d \mu^{\prime} d \varphi^{\prime}
$$

November 28, 1995 
for $\mu \in(0,1]$ and $\varphi \in[0,2 \pi]$.

As we wish to include the possibility that there could be some phenomenon (e.g. rows of plants, or ocean waves) related to the ground that introduces a special direction into the problem, we make no assumptions here regarding the symmetry of the reflection function $R\left(\mu^{\prime}, \mu, \varphi^{\prime}, \varphi\right)$. We note that, in writing the boundary condition at the ground as we have in Eq. (3b), we are considering that the reflection function describes the reflection of radiation from some direction defined by the variables $\left(\mu^{\prime}, \varphi^{\prime}\right)$, with $\mu^{\prime} \in(0,1]$ and $\varphi^{\prime} \in[0,2 \pi]$, to another direction defined by the variables $(-\mu, \varphi)$, with $\mu \in(0,1]$ and $\varphi \in[0,2 \pi]$.

\section{The Unscattered Component of the Intensity}

Since the incident beam for the considered problem is represented by delta functions, the resulting intensity will also have a component containing generalized functions, and so, in order to avoid the impossible task of trying to compute generalized functions, we use first a decomposition of the form

$$
I(\tau, \mu, \varphi)=I_{0}(\tau, \mu, \varphi)+I_{*}(\tau, \mu, \varphi)
$$

where $I_{0}(\tau, \mu, \varphi)$ denotes the solution of Eqs. (1) and (3) for the case $\varpi=0$ and where $I_{*}(\tau, \mu, \varphi)$ is the complementary component of the solution. We note that even if the reflection function at the ground has a component for specular reflection, the decomposition given by Eq. (4) is such that all of the generalized functions in the solution $I(\tau, \mu, \varphi)$ will be contained in $I_{0}(\tau, \mu, \varphi)$.

Solving Eqs. (1) and (3) for the case $\varpi=0$, we find

$$
I_{0}(\tau, \mu, \varphi)=\pi \delta\left(\mu-\mu_{0}\right) \delta\left(\varphi-\varphi_{0}\right) e^{-\tau / \mu}
$$

and

$$
I_{0}(\tau,-\mu, \varphi)=\pi \mu_{0} R\left(\mu_{0}, \mu, \varphi_{0}, \varphi\right) e^{-\tau_{0} / \mu_{0}} e^{-\left(\tau_{0}-\tau\right) / \mu}
$$

for $\mu \in(0,1]$ and $\varphi \in[0,2 \pi]$.

\section{The Complementary Component of the Intensity}

We can now substitute Eq. (4) into Eqs. (1) and (3) and deduce, after noting Eqs. (5), that the complementary component $I_{*}(\tau, \mu, \varphi)$ is defined by

$$
\mu \frac{\partial}{\partial \tau} I_{*}(\tau, \mu, \varphi)+I_{*}(\tau, \mu, \varphi)=\frac{\varpi}{4 \pi} \int_{-1}^{1} \int_{0}^{2 \pi} p(\cos \Theta) I_{*}\left(\tau, \mu^{\prime}, \varphi^{\prime}\right) d \varphi^{\prime} d \mu^{\prime}+F(\tau, \mu, \varphi)
$$

for $\tau \in\left(0, \tau_{0}\right), \mu \in[-1,1]$ and $\varphi \in[0,2 \pi]$, and the boundary conditions

$$
I_{*}(0, \mu, \varphi)=0
$$

November 28, 1995 
and

$$
I_{*}\left(\tau_{0},-\mu, \varphi\right)=\int_{0}^{2 \pi} \int_{0}^{1} R\left(\mu^{\prime}, \mu, \varphi^{\prime}, \varphi\right) I_{*}\left(\tau_{0}, \mu^{\prime}, \varphi^{\prime}\right) \mu^{\prime} d \mu^{\prime} d \varphi^{\prime}
$$

for $\mu \in(0,1]$ and $\varphi \in[0,2 \pi]$. Here, the known inhomogeneous term is

$$
F(\tau, \mu, \varphi)=\frac{\varpi}{4 \pi} \int_{-1}^{1} \int_{0}^{2 \pi} p(\cos \Theta) I_{0}\left(\tau, \mu^{\prime}, \varphi^{\prime}\right) d \varphi^{\prime} d \mu^{\prime}
$$

Before attempting a Fourier decomposition of the complementary component of the intensity, we make use of the addition theorem ${ }^{3}$ for the Legendre polynomials and express the scattering law as

$$
p(\cos \Theta)=\sum_{m=0}^{L}\left(2-\delta_{0, m}\right) \sum_{l=m}^{L} \beta_{l}^{m} P_{l}^{m}\left(\mu^{\prime}\right) P_{l}^{m}(\mu) \cos \left[m\left(\varphi^{\prime}-\varphi\right)\right]
$$

where

$$
P_{l}^{m}(\mu)=\left(1-\mu^{2}\right)^{m / 2} \frac{d^{m}}{d \mu^{m}} P_{l}(\mu)
$$

denotes an associated Legendre function, and where

$$
\beta_{l}^{m}=\frac{(l-m) !}{(l+m) !} \beta_{l}
$$

Using Eqs. (5) and (9), we rewrite Eq. (6) as

$$
\begin{aligned}
\mu \frac{\partial}{\partial \tau} I_{*}(\tau, \mu, \varphi)+I_{*}(\tau, \mu, \varphi)=\frac{\varpi}{4 \pi} \sum_{m=0}^{L}\left(2-\delta_{0, m}\right) \sum_{l=m}^{L} \beta_{l}^{m} P_{l}^{m}(\mu) \\
\quad \times \int_{-1}^{1} \int_{0}^{2 \pi} P_{l}^{m}\left(\mu^{\prime}\right) I_{*}\left(\tau, \mu^{\prime}, \varphi^{\prime}\right) \cos \left[m\left(\varphi^{\prime}-\varphi\right)\right] d \varphi^{\prime} d \mu^{\prime}+F(\tau, \mu, \varphi)
\end{aligned}
$$

where now we write the inhomogeneous term as

$$
F(\tau, \mu, \varphi)=F_{1}(\tau, \mu, \varphi)+F_{2}(\tau, \mu, \varphi)
$$

with

$$
F_{1}(\tau, \mu, \varphi)=\frac{\varpi}{4} \sum_{m=0}^{L}\left(2-\delta_{0, m}\right) \sum_{l=m}^{L} \beta_{l}^{m} P_{l}^{m}\left(\mu_{0}\right) P_{l}^{m}(\mu) \cos \left[m\left(\varphi-\varphi_{0}\right)\right] e^{-\tau / \mu_{0}}
$$

and

$$
\begin{aligned}
F_{2}(\tau, \mu, \varphi)=\frac{\varpi}{4} \mu_{0} \sum_{m=0}^{L}\left(2-\delta_{0, m}\right) & \sum_{l=m}^{L} \beta_{l}^{m} P_{l}^{m}(\mu)(-1)^{l-m} e^{-\tau_{0} / \mu_{0}} \\
& \times \int_{0}^{1} \int_{0}^{2 \pi} P_{l}^{m}\left(\mu^{\prime}\right) R\left(\mu_{0}, \mu^{\prime}, \varphi_{0}, \varphi^{\prime}\right) \cos \left[m\left(\varphi^{\prime}-\varphi\right)\right] e^{-\left(\tau_{0}-\tau\right) / \mu^{\prime}} d \varphi^{\prime} d \mu^{\prime}
\end{aligned}
$$

November 28, 1995 
In order to simplify Eq. (14b), we first let $\varphi_{r}$ denote a reference direction, and then we expand the reflection function in a Fourier series of the form

$$
R\left(\mu^{\prime}, \mu, \varphi^{\prime}, \varphi\right)=\frac{1}{2} \sum_{n=0}^{\infty}\left(2-\delta_{0, n}\right) R_{n}^{c}\left(\mu^{\prime}, \mu, \varphi^{\prime}\right) \cos \left[n\left(\varphi-\varphi_{r}\right)\right]+\sum_{n=1}^{\infty} R_{n}^{s}\left(\mu^{\prime}, \mu, \varphi^{\prime}\right) \sin \left[n\left(\varphi-\varphi_{r}\right)\right],
$$

where, of course,

$$
R_{n}^{c}\left(\mu^{\prime}, \mu, \varphi^{\prime}\right)=\frac{1}{\pi} \int_{0}^{2 \pi} R\left(\mu^{\prime}, \mu, \varphi^{\prime}, \varphi\right) \cos \left[n\left(\varphi-\varphi_{r}\right)\right] d \varphi, \quad n \geq 0
$$

and

$$
R_{n}^{s}\left(\mu^{\prime}, \mu, \varphi^{\prime}\right)=\frac{1}{\pi} \int_{0}^{2 \pi} R\left(\mu^{\prime}, \mu, \varphi^{\prime}, \varphi\right) \sin \left[n\left(\varphi-\varphi_{r}\right)\right] d \varphi, \quad n \geq 1 .
$$

Since later in this work we will use a double Fourier series representation of the reflection function, we also write here

$$
R_{n}^{c}\left(\mu^{\prime}, \mu, \varphi^{\prime}\right)=\frac{1}{2} \sum_{m=0}^{\infty}\left(2-\delta_{0, m}\right) R_{n, m}^{c c}\left(\mu^{\prime}, \mu\right) \cos \left[m\left(\varphi^{\prime}-\varphi_{r}\right)\right]+\sum_{m=1}^{\infty} R_{n, m}^{c s}\left(\mu^{\prime}, \mu\right) \sin \left[m\left(\varphi^{\prime}-\varphi_{r}\right)\right]
$$

and

$$
R_{n}^{s}\left(\mu^{\prime}, \mu, \varphi^{\prime}\right)=\frac{1}{2} \sum_{m=0}^{\infty}\left(2-\delta_{0, m}\right) R_{n, m}^{s c}\left(\mu^{\prime}, \mu\right) \cos \left[m\left(\varphi^{\prime}-\varphi_{r}\right)\right]+\sum_{m=1}^{\infty} R_{n, m}^{s s}\left(\mu^{\prime}, \mu\right) \sin \left[m\left(\varphi^{\prime}-\varphi_{r}\right)\right]
$$

with

$$
\begin{array}{ll}
R_{n, m}^{c c}\left(\mu^{\prime}, \mu\right)=\frac{1}{\pi} \int_{0}^{2 \pi} R_{n}^{c}\left(\mu^{\prime}, \mu, \varphi^{\prime}\right) \cos \left[m\left(\varphi^{\prime}-\varphi_{r}\right)\right] d \varphi^{\prime}, & n \geq 0, m \geq 0, \\
R_{n, m}^{c s}\left(\mu^{\prime}, \mu\right)=\frac{1}{\pi} \int_{0}^{2 \pi} R_{n}^{c}\left(\mu^{\prime}, \mu, \varphi^{\prime}\right) \sin \left[m\left(\varphi^{\prime}-\varphi_{r}\right)\right] d \varphi^{\prime}, & n \geq 0, m \geq 1, \\
R_{n, m}^{s c}\left(\mu^{\prime}, \mu\right)=\frac{1}{\pi} \int_{0}^{2 \pi} R_{n}^{s}\left(\mu^{\prime}, \mu, \varphi^{\prime}\right) \cos \left[m\left(\varphi^{\prime}-\varphi_{r}\right)\right] d \varphi^{\prime}, & n \geq 1, m \geq 0,
\end{array}
$$

and

$$
R_{n, m}^{s s}\left(\mu^{\prime}, \mu\right)=\frac{1}{\pi} \int_{0}^{2 \pi} R_{n}^{s}\left(\mu^{\prime}, \mu, \varphi^{\prime}\right) \sin \left[m\left(\varphi^{\prime}-\varphi_{r}\right)\right] d \varphi^{\prime}, \quad n \geq 1, m \geq 1 .
$$

Substituting Eq. (15) into Eq. (14b), we find

$$
F_{2}(\tau, \mu, \varphi)=\frac{1}{2} \sum_{m=0}^{L}\left(2-\delta_{0, m}\right) F_{2, m}^{c}(\tau, \mu) \cos \left[m\left(\varphi-\varphi_{r}\right)\right]+\sum_{m=1}^{L} F_{2, m}^{s}(\tau, \mu) \sin \left[m\left(\varphi-\varphi_{r}\right)\right]
$$

where

$$
F_{2, m}^{c}(\tau, \mu)=\frac{\varpi \pi}{2} \mu_{0} \sum_{l=m}^{L} \beta_{l}^{m} P_{l}^{m}(\mu)(-1)^{l-m} e^{-\tau_{0} / \mu_{0}} \int_{0}^{1} P_{l}^{m}\left(\mu^{\prime}\right) R_{m}^{c}\left(\mu_{0}, \mu^{\prime}, \varphi_{0}\right) e^{-\left(\tau_{0}-\tau\right) / \mu^{\prime}} d \mu^{\prime}
$$

November 28, 1995 
and

$$
F_{2, m}^{s}(\tau, \mu)=\frac{\varpi \pi}{2} \mu_{0} \sum_{l=m}^{L} \beta_{l}^{m} P_{l}^{m}(\mu)(-1)^{l-m} e^{-\tau_{0} / \mu_{0}} \int_{0}^{1} P_{l}^{m}\left(\mu^{\prime}\right) R_{m}^{s}\left(\mu_{0}, \mu^{\prime}, \varphi_{0}\right) e^{-\left(\tau_{0}-\tau\right) / \mu^{\prime}} d \mu^{\prime} .
$$

By defining

$$
F_{1, m}^{c}(\tau, \mu)=\frac{\varpi}{2} \sum_{l=m}^{L} \beta_{l}^{m} P_{l}^{m}\left(\mu_{0}\right) P_{l}^{m}(\mu) \cos \left[m\left(\varphi_{0}-\varphi_{r}\right)\right] e^{-\tau / \mu_{0}}
$$

and

$$
F_{1, m}^{s}(\tau, \mu)=\frac{\varpi}{2} \sum_{l=m}^{L} \beta_{l}^{m} P_{l}^{m}\left(\mu_{0}\right) P_{l}^{m}(\mu) \sin \left[m\left(\varphi_{0}-\varphi_{r}\right)\right] e^{-\tau / \mu_{0}},
$$

we can rewrite Eq. (14a) as

$$
F_{1}(\tau, \mu, \varphi)=\frac{1}{2} \sum_{m=0}^{L}\left(2-\delta_{0, m}\right) F_{1, m}^{c}(\tau, \mu) \cos \left[m\left(\varphi-\varphi_{r}\right)\right]+\sum_{m=1}^{L} F_{1, m}^{s}(\tau, \mu) \sin \left[m\left(\varphi-\varphi_{r}\right)\right]
$$

Now, with

$$
F_{m}^{c}(\tau, \mu)=F_{1, m}^{c}(\tau, \mu)+F_{2, m}^{c}(\tau, \mu)
$$

and

$$
F_{m}^{s}(\tau, \mu)=F_{1, m}^{s}(\tau, \mu)+F_{2, m}^{s}(\tau, \mu)
$$

we can write the inhomogeneous term in Eq. (12) as

$$
F(\tau, \mu, \varphi)=\frac{1}{2} \sum_{m=0}^{L}\left(2-\delta_{0, m}\right) F_{m}^{c}(\tau, \mu) \cos \left[m\left(\varphi-\varphi_{r}\right)\right]+\sum_{m=1}^{L} F_{m}^{s}(\tau, \mu) \sin \left[m\left(\varphi-\varphi_{r}\right)\right] .
$$

Considering the use of Fourier series again, we substitute

$$
I_{*}(\tau, \mu, \varphi)=\frac{1}{2} \sum_{m=0}^{\infty}\left(2-\delta_{0, m}\right) I_{*, m}^{c}(\tau, \mu) \cos \left[m\left(\varphi-\varphi_{r}\right)\right]+\sum_{m=1}^{\infty} I_{*, m}^{s}(\tau, \mu) \sin \left[m\left(\varphi-\varphi_{r}\right)\right]
$$

into Eq. (12) and note Eq. (24) to find, for $m=0,1, \ldots L$,

$$
\mu \frac{\partial}{\partial \tau} I_{*, m}^{c}(\tau, \mu)+I_{*, m}^{c}(\tau, \mu)=\frac{\varpi}{2} \sum_{l=m}^{L} \beta_{l}^{m} P_{l}^{m}(\mu) \int_{-1}^{1} P_{l}^{m}\left(\mu^{\prime}\right) I_{*, m}^{c}\left(\tau, \mu^{\prime}\right) d \mu^{\prime}+F_{m}^{c}(\tau, \mu)
$$

and, for $m=1,2, \ldots L$,

$$
\mu \frac{\partial}{\partial \tau} I_{*, m}^{s}(\tau, \mu)+I_{*, m}^{s}(\tau, \mu)=\frac{\varpi}{2} \sum_{l=m}^{L} \beta_{l}^{m} P_{l}^{m}(\mu) \int_{-1}^{1} P_{l}^{m}\left(\mu^{\prime}\right) I_{*, m}^{s}\left(\tau, \mu^{\prime}\right) d \mu^{\prime}+F_{m}^{s}(\tau, \mu) .
$$

In addition, we find, for $m=L+1, L+2, \ldots$,

$$
\mu \frac{\partial}{\partial \tau} I_{*, m}^{c}(\tau, \mu)+I_{*, m}^{c}(\tau, \mu)=0
$$

November 28, 1995 
and

$$
\mu \frac{\partial}{\partial \tau} I_{*, m}^{s}(\tau, \mu)+I_{*, m}^{s}(\tau, \mu)=0
$$

Finally we substitute Eqs. (15), (17) and (25) into Eqs. (7) and deduce the required boundary conditions on the Fourier components of $I_{*}(\tau, \mu, \varphi)$. We find from Eq. (7a)

$$
I_{*, m}^{c}(0, \mu)=0, \quad m=0,1,2, \ldots
$$

and

$$
I_{*, m}^{s}(0, \mu)=0, \quad m=1,2,3, \ldots
$$

for $\mu \in(0,1]$. From Eq. (7b) we find, for $m=0,1,2, \ldots$ and $\mu \in(0,1]$,

$$
I_{*, m}^{c}\left(\tau_{0},-\mu\right)=\frac{\pi}{2} \sum_{n=0}^{\infty}\left(2-\delta_{0, n}\right) \int_{0}^{1} R_{m, n}^{c c}\left(\mu^{\prime}, \mu\right) I_{*, n}^{c}\left(\tau_{0}, \mu^{\prime}\right) \mu^{\prime} d \mu^{\prime}+\pi \sum_{n=1}^{\infty} \int_{0}^{1} R_{m, n}^{c s}\left(\mu^{\prime}, \mu\right) I_{*, n}^{s}\left(\tau_{0}, \mu^{\prime}\right) \mu^{\prime} d \mu^{\prime}
$$

and, for $m=1,2,3, \ldots$ and $\mu \in(0,1]$,

$$
I_{*, m}^{s}\left(\tau_{0},-\mu\right)=\frac{\pi}{2} \sum_{n=0}^{\infty}\left(2-\delta_{0, n}\right) \int_{0}^{1} R_{m, n}^{s c}\left(\mu^{\prime}, \mu\right) I_{*, n}^{c}\left(\tau_{0}, \mu^{\prime}\right) \mu^{\prime} d \mu^{\prime}+\pi \sum_{n=1}^{\infty} \int_{0}^{1} R_{m, n}^{s s}\left(\mu^{\prime}, \mu\right) I_{*, n}^{s}\left(\tau_{0}, \mu^{\prime}\right) \mu^{\prime} d \mu^{\prime}
$$

Considering now the component problems for $m>L$, we can solve Eqs. (27) subject to the conditions of Eqs. (28) to find

$$
I_{*, m}^{c}(\tau, \mu)=0
$$

and

$$
I_{*, m}^{s}(\tau, \mu)=0
$$

for $\mu \in(0,1]$. We also find

$$
I_{*, m}^{c}(\tau,-\mu)=I_{*, m}^{c}\left(\tau_{0},-\mu\right) e^{-\left(\tau_{0}-\tau\right) / \mu}
$$

and

$$
I_{*, m}^{s}(\tau,-\mu)=I_{*, m}^{s}\left(\tau_{0},-\mu\right) e^{-\left(\tau_{0}-\tau\right) / \mu}
$$

for $\mu \in(0,1]$. Here $I_{*, m}^{c}\left(\tau_{0},-\mu\right)$ and $I_{*, m}^{s}\left(\tau_{0},-\mu\right)$ are to be determined.

Noting Eqs. (30), we see that the summations in Eqs. (29) truncate, so we write the boundary conditions at $\tau=\tau_{0}$ as

$$
I_{*, m}^{c}\left(\tau_{0},-\mu\right)=\frac{\pi}{2} \sum_{n=0}^{L}\left(2-\delta_{0, n}\right) \int_{0}^{1} R_{m, n}^{c c}\left(\mu^{\prime}, \mu\right) I_{*, n}^{c}\left(\tau_{0}, \mu^{\prime}\right) \mu^{\prime} d \mu^{\prime}+\pi \sum_{n=1}^{L} \int_{0}^{1} R_{m, n}^{c s}\left(\mu^{\prime}, \mu\right) I_{*, n}^{s}\left(\tau_{0}, \mu^{\prime}\right) \mu^{\prime} d \mu^{\prime}
$$

November 28, 1995 
for $m \geq 0$ and $\mu \in(0,1]$, and

$$
I_{*, m}^{s}\left(\tau_{0},-\mu\right)=\frac{\pi}{2} \sum_{n=0}^{L}\left(2-\delta_{0, n}\right) \int_{0}^{1} R_{m, n}^{s c}\left(\mu^{\prime}, \mu\right) I_{*, n}^{c}\left(\tau_{0}, \mu^{\prime}\right) \mu^{\prime} d \mu^{\prime}+\pi \sum_{n=1}^{L} \int_{0}^{1} R_{m, n}^{s s}\left(\mu^{\prime}, \mu\right) I_{*, n}^{s}\left(\tau_{0}, \mu^{\prime}\right) \mu^{\prime} d \mu^{\prime},
$$

for $m \geq 1$ and $\mu \in(0,1]$. It follows that once we have solved our basic problems for $m=0,1,2, \ldots L$, we can compute the functions required to complete the solutions given by Eqs. (30) and (31) from Eqs. (32) with $m>L$.

\section{Special Cases}

At this point we would like to show how the foregoing development reduces to simpler forms for three special cases. We consider first a mixture of specular and diffuse reflection. We thus write

$$
R\left(\mu^{\prime}, \mu, \varphi^{\prime}, \varphi\right)=\left(\rho_{s} / \mu\right) \delta\left(\mu^{\prime}-\mu\right) \delta\left(\varphi^{\prime}-\varphi\right)+\rho_{d} / \pi
$$

where $\rho_{s}$ and $\rho_{d}$ are, respectively, the coefficients for specular and diffuse reflection. In this (classic) example of reflection, there clearly is no preferred direction on the ground, and so we are free to choose $\varphi_{r}=\varphi_{0}$. Looking back to Eq. (5b), we find we can now write

$$
I_{0}(\tau,-\mu, \varphi)=\left[\pi \rho_{s} \delta\left(\mu-\mu_{0}\right) \delta\left(\varphi-\varphi_{0}\right)+\mu_{0} \rho_{d}\right] e^{-\tau_{0} / \mu_{0}} e^{-\left(\tau_{0}-\tau\right) / \mu}
$$

for $\mu \in(0,1]$. We next observe that for this special case all sine components of $I_{*}(\tau, \mu, \varphi)$ are zero. In addition, Eq. (21a) reduces to

$$
F_{1, m}^{c}(\tau, \mu)=\frac{\varpi}{2} \sum_{l=m}^{L} \beta_{l}^{m} P_{l}^{m}\left(\mu_{0}\right) P_{l}^{m}(\mu) e^{-\tau / \mu_{0}}
$$

and Eq. (20a) becomes

$$
\begin{aligned}
F_{2, m}^{c}(\tau, \mu)=\frac{\varpi}{2} \rho_{s} \sum_{l=m}^{L} \beta_{l}^{m} P_{l}^{m}\left(\mu_{0}\right) P_{l}^{m}(\mu)(-1)^{l-m} e^{-\left(2 \tau_{0}-\tau\right) / \mu_{0}} & \\
& +\varpi \mu_{0} \rho_{d} \delta_{0, m} \sum_{l=0}^{L} \beta_{l} P_{l}(\mu)(-1)^{l} e^{-\tau_{0} / \mu_{0}} \int_{0}^{1} P_{l}\left(\mu^{\prime}\right) e^{-\left(\tau_{0}-\tau\right) / \mu^{\prime}} d \mu^{\prime} .
\end{aligned}
$$

It follows that, with Eqs. (35) and

$$
F_{m}^{c}(\tau, \mu)=F_{1, m}^{c}(\tau, \mu)+F_{2, m}^{c}(\tau, \mu)
$$

the inhomogeneous source term in Eq. (26a) is explicitly available. We can now use Eq. (33) in Eqs. (16a) and $(18 \mathrm{a})$ to find

$$
R_{m, n}^{c c}\left(\mu^{\prime}, \mu\right)=\frac{1}{\pi}\left(1+\delta_{0, n}\right) \delta_{m, n}\left[\left(\rho_{s} / \mu\right) \delta\left(\mu^{\prime}-\mu\right)+2 \rho_{d} \delta_{0, n}\right], \quad m \geq 0, n \geq 0
$$


so that we can conclude, from Eqs. (28a) and (32a), that the boundary conditions subject to which we must solve Eq. (26a), for $m=0,1,2, \ldots L$, are

$$
I_{*, m}^{c}(0, \mu)=0
$$

and

$$
I_{*, m}^{c}\left(\tau_{0},-\mu\right)=\rho_{s} I_{*, m}^{c}\left(\tau_{0}, \mu\right)+2 \rho_{d} \delta_{0, m} \int_{0}^{1} I_{*, 0}^{c}\left(\tau_{0}, \mu^{\prime}\right) \mu^{\prime} d \mu^{\prime}
$$

for $\mu \in(0,1]$. It is clear from Eqs. (30a), (31a), (32a) and (37) that $I_{*, m}^{c}(\tau, \mu)=0$ for $m>L$.

For a second special case, we consider a reflection function of the form

$$
R\left(\mu^{\prime}, \mu, \varphi^{\prime}, \varphi\right)=\sum_{k=0}^{K} b_{k} P_{k}(\cos \Xi)
$$

where $\Xi$ is the angle between the vectors that define the incident and emergent directions of the radiation. In addition, the $\left\{b_{k}\right\}$ are coefficients that are assumed known. As we did with the phase function, we can use the addition theorem ${ }^{3}$ for the Legendre polynomials to rewrite Eq. (39) as

$$
R\left(\mu^{\prime}, \mu, \varphi^{\prime}, \varphi\right)=\sum_{j=0}^{K}\left(2-\delta_{0, j}\right) \sum_{k=j}^{K}(-1)^{k-j} b_{k}^{j} P_{k}^{j}\left(\mu^{\prime}\right) P_{k}^{j}(\mu) \cos \left[j\left(\varphi^{\prime}-\varphi\right)\right]
$$

where

$$
b_{k}^{j}=\frac{(k-j) !}{(k+j) !} b_{k} .
$$

We note that if we choose $\varphi_{r}=\varphi_{0}$ then the complementary part of the intensity $I_{*}(\tau, \mu, \varphi)$ will not have any sine components. Here again Eq. (21a) reduces to

$$
F_{1, m}^{c}(\tau, \mu)=\frac{\varpi}{2} \sum_{l=m}^{L} \beta_{l}^{m} P_{l}^{m}\left(\mu_{0}\right) P_{l}^{m}(\mu) e^{-\tau / \mu_{0}}
$$

and Eq. (20a) becomes

$$
\begin{aligned}
F_{2, m}^{c}(\tau, \mu)=\varpi \pi \mu_{0} \sum_{l=m}^{L} \beta_{l}^{m} P_{l}^{m}(\mu)(-1)^{l-m} e^{-\tau_{0} / \mu_{0}} \sum_{k=m}^{K}(-1)^{k-m} b_{k}^{m} P_{k}^{m}\left(\mu_{0}\right) & \\
& \times \int_{0}^{1} P_{l}^{m}\left(\mu^{\prime}\right) P_{k}^{m}\left(\mu^{\prime}\right) e^{-\left(\tau_{0}-\tau\right) / \mu^{\prime}} d \mu^{\prime} .
\end{aligned}
$$

We find that, with Eqs. (42) and

$$
F_{m}^{c}(\tau, \mu)=F_{1, m}^{c}(\tau, \mu)+F_{2, m}^{c}(\tau, \mu)
$$

the inhomogeneous source term in Eq. (26a) is again explicitly available. Upon using Eq. (40) in Eqs. (16a) and (18a), we find

$$
R_{m, n}^{c c}\left(\mu^{\prime}, \mu\right)=2\left(1+\delta_{0, n}\right) \delta_{m, n} \sum_{k=n}^{K}(-1)^{k-n} b_{k}^{n} P_{k}^{n}\left(\mu^{\prime}\right) P_{k}^{n}(\mu), \quad m \geq 0, n \geq 0 .
$$


It now follows, from Eqs. (28a) and (32a), that the boundary conditions subject to which we must solve Eq. (26a), for $m=0,1,2, \ldots L$, are

$$
I_{*, m}^{c}(0, \mu)=0
$$

and

$$
I_{*, m}^{c}\left(\tau_{0},-\mu\right)=\int_{0}^{1} T_{m}\left(\mu^{\prime}, \mu\right) I_{*, m}^{c}\left(\tau_{0}, \mu^{\prime}\right) \mu^{\prime} d \mu^{\prime}
$$

for $\mu \in(0,1]$. Here

$$
T_{m}\left(\mu^{\prime}, \mu\right)=2 \pi \sum_{k=m}^{K}(-1)^{k-m} b_{k}^{m} P_{k}^{m}\left(\mu^{\prime}\right) P_{k}^{m}(\mu) .
$$

To complete the formulation for this special case, we note that $I_{*, m}^{c}(\tau, \mu)=0$ for $m>L$.

Our third (and last) special case is defined by the reflection function

$$
R\left(\mu^{\prime}, \mu, \varphi^{\prime}, \varphi\right)=\frac{1}{2} \sum_{k=0}^{K_{c}}\left(2-\delta_{0, k}\right) a_{k}\left(\mu^{\prime}, \mu\right) \cos \left[k\left(\varphi^{\prime}-\varphi\right)\right]+\sum_{k=1}^{K_{s}} b_{k}\left(\mu^{\prime}, \mu\right) \sin \left[k\left(\varphi^{\prime}-\varphi\right)\right]
$$

where the coefficients $\left\{a_{k}\left(\mu^{\prime}, \mu\right)\right\}$ and $\left\{b_{k}\left(\mu^{\prime}, \mu\right)\right\}$ are assumed known. We note that Eq. (47) covers the cases studied by Godsalve ${ }^{1}$ and by Walthall et al., ${ }^{4}$ as well as the scalar problem obtained by neglecting polarization effects in the formulation of Deuzé, Herman and Santer. ${ }^{5}$

Here again the reflection function does not involve a preferred direction so we can set $\varphi_{r}=\varphi_{0}$. With this choice of $\varphi_{r}$, Eqs. (21) become

$$
F_{1, m}^{c}(\tau, \mu)=\frac{\varpi}{2} \sum_{l=m}^{L} \beta_{l}^{m} P_{l}^{m}\left(\mu_{0}\right) P_{l}^{m}(\mu) e^{-\tau / \mu_{0}}
$$

and

$$
F_{1, m}^{s}(\tau, \mu)=0
$$

In addition, after noting that Eqs. (16) yield, for this special case,

$$
\begin{gathered}
R_{0}^{c}\left(\mu^{\prime}, \mu, \varphi^{\prime}\right)=a_{0}\left(\mu^{\prime}, \mu\right) \\
R_{n}^{c}\left(\mu^{\prime}, \mu, \varphi^{\prime}\right)=a_{n}\left(\mu^{\prime}, \mu\right) \cos \left[n\left(\varphi^{\prime}-\varphi_{0}\right)\right]+b_{n}\left(\mu^{\prime}, \mu\right) \sin \left[n\left(\varphi^{\prime}-\varphi_{0}\right)\right]
\end{gathered}
$$

and

$$
R_{n}^{s}\left(\mu^{\prime}, \mu, \varphi^{\prime}\right)=a_{n}\left(\mu^{\prime}, \mu\right) \sin \left[n\left(\varphi^{\prime}-\varphi_{0}\right)\right]-b_{n}\left(\mu^{\prime}, \mu\right) \cos \left[n\left(\varphi^{\prime}-\varphi_{0}\right)\right]
$$

for $n \geq 1$, we write Eqs. (20) as

$$
F_{2, m}^{c}(\tau, \mu)=\frac{\varpi \pi}{2} \mu_{0} \sum_{l=m}^{L} \beta_{l}^{m} P_{l}^{m}(\mu)(-1)^{l-m} e^{-\tau_{0} / \mu_{0}} \int_{0}^{1} P_{l}^{m}\left(\mu^{\prime}\right) a_{m}\left(\mu_{0}, \mu^{\prime}\right) e^{-\left(\tau_{0}-\tau\right) / \mu^{\prime}} d \mu^{\prime},
$$

November 28, 1995 
for $m \geq 0$, and

$$
F_{2, m}^{s}(\tau, \mu)=-\frac{\varpi \pi}{2} \mu_{0} \sum_{l=m}^{L} \beta_{l}^{m} P_{l}^{m}(\mu)(-1)^{l-m} e^{-\tau_{0} / \mu_{0}} \int_{0}^{1} P_{l}^{m}\left(\mu^{\prime}\right) b_{m}\left(\mu_{0}, \mu^{\prime}\right) e^{-\left(\tau_{0}-\tau\right) / \mu^{\prime}} d \mu^{\prime}
$$

for $m \geq 1$. From Eqs. (50), it is clear that, for this case, we must solve both the sine and cosine component problems defined by Eqs. (26), with the corresponding inhomogeneous source terms given explicitly by Eqs. (23), (48) and (50). In regard to the boundary conditions at the ground, we can use the expressions

$$
\begin{gathered}
R_{m, n}^{c c}\left(\mu^{\prime}, \mu\right)=\left(1+\delta_{0, n}\right) \delta_{m, n} a_{n}\left(\mu^{\prime}, \mu\right), \quad m \geq 0, n \geq 0, \\
R_{m, n}^{c s}\left(\mu^{\prime}, \mu\right)=\delta_{m, n} b_{n}\left(\mu^{\prime}, \mu\right), \quad m \geq 0, n \geq 1 \\
R_{m, 0}^{s c}\left(\mu^{\prime}, \mu\right)=0, \quad m \geq 1, \\
R_{m, n}^{s c}\left(\mu^{\prime}, \mu\right)=-\delta_{m, n} b_{n}\left(\mu^{\prime}, \mu\right), \quad m \geq 1, n \geq 1,
\end{gathered}
$$

and

$$
R_{m, n}^{s s}\left(\mu^{\prime}, \mu\right)=\delta_{m, n} a_{n}\left(\mu^{\prime}, \mu\right), \quad m \geq 1, n \geq 1
$$

obtained by substituting Eqs. (49) into Eqs. (18), to find that Eqs. (32) reduce to

$$
I_{*, 0}^{c}\left(\tau_{0},-\mu\right)=\pi \int_{0}^{1} a_{0}\left(\mu^{\prime}, \mu\right) I_{*, 0}^{c}\left(\tau_{0}, \mu^{\prime}\right) \mu^{\prime} d \mu^{\prime}
$$

for $\mu \in(0,1]$,

$$
I_{*, m}^{c}\left(\tau_{0},-\mu\right)=\pi \int_{0}^{1}\left[a_{m}\left(\mu^{\prime}, \mu\right) I_{*, m}^{c}\left(\tau_{0}, \mu^{\prime}\right)+b_{m}\left(\mu^{\prime}, \mu\right) I_{*, m}^{s}\left(\tau_{0}, \mu^{\prime}\right)\right] \mu^{\prime} d \mu^{\prime}
$$

and

$$
I_{*, m}^{s}\left(\tau_{0},-\mu\right)=\pi \int_{0}^{1}\left[a_{m}\left(\mu^{\prime}, \mu\right) I_{*, m}^{s}\left(\tau_{0}, \mu^{\prime}\right)-b_{m}\left(\mu^{\prime}, \mu\right) I_{*, m}^{c}\left(\tau_{0}, \mu^{\prime}\right)\right] \mu^{\prime} d \mu^{\prime},
$$

for $m \geq 1$ and $\mu \in(0,1]$. Finally, we complete the formulation for this case by noting that $I_{*, m}^{c}(\tau, \mu)=0$ for $m>L$ and that $I_{*, m}^{s}(\tau, \mu)=0$ for $m>\min \left\{L, K_{s}\right\}$.

\section{Concluding Remarks}

Having considered a fairly general reflection function, we find that the Fourier decomposition is considerably more complicated than what we are accustomed to seeing in radiative transfer calculations in plane geometry. In particular, we see that for the general case an infinite number of sine and cosine components of $I_{*}(\tau, \mu, \varphi)$ are required to establish the solution. We note also that the first $L$ sine and the first $L+1$ cosine components of $I_{*}(\tau, \mu, \varphi)$ are coupled by the boundary condition at the ground and that the solution 
for the remaining sine and cosine components of $I_{*}(\tau, \mu, \varphi)$ can be expressed in terms of the solutions for those $2 L+1$ components. Needless to say, some simplifying assumptions about the reflection function, as, for example, the ones we have made in Section 4, could make the formulation of the desired solution for the intensity a great deal more tractable.

\section{Acknowledgements}

One of the authors (L.B.B.) would like to express her thanks to the Mathematics Department of North Carolina State University for the kind hospitality extended throughout a period during which this work was

done. In addition, it is noted that the work of L.B.B. and R.D.M.G. was supported in part by CNPq of Brasil.

\section{References}

1. C. Godsalve, JQSRT 53, 289 (1995).

2. S. Chandrasekhar, Radiative Transfer, Oxford University Press, London (1950).

3. I. S. Gradshteyn and I. M. Ryzhik, Table of Integrals, Series, and Products, Academic Press, New York, NY (1980).

4. C. L. Walthall, J. M. Norman, J. M. Welles, G. Campbell and B. L. Blad, Applied Optics $\mathbf{2 4}, 383(1985)$.

5. J. L. Deuzé, M. Herman and R. Santer, JQSRT 41, 483 (1989). 